\title{
Colloidal nanocrystal synthesis and the organic-inorganic interface
}

Yadong Yin \& A. Paul Alivisatos

Department of Chemistry, University of California, Berkeley and the Molecular Foundry, Lawrence Berkeley National Laboratory, Berkeley, CA 94720, USA

(email: alivis@berkeley.edu)

Colloidal nanocrystals are nanometer-sized, solution-grown inorganic particles stabilized by a layer of surfactants attached to their surface. The inorganic cores exhibit useful properties controlled by composition as well as size and shape, while the surfactant coating ensures that these structures are easy to fabricate and process. It is this combination of features that makes colloidal nanocrystals attractive and promising building blocks for advanced materials and devices. But their full potential can only be exploited if we achieve exquisite control over their composition, size, shape, crystal structure and surface properties. Here we review what is known about nanocrystal growth and outline strategies for controlling it.

Colloidal nanocrystals are sometimes referred to as 'artificial atoms' because the density of their electronic states - which controls many physical properties-can be widely and easily tuned by adjusting composition, size and shape. The combination of strongly size- and shape-dependent physical properties and ease of fabrication and processing makes nanocrystals promising building blocks for materials with designed functions $^{1,2}$. But the ability to control the uniformity of the size, shape, composition, crystal structure and surface properties of the nanocrystals is not only of technological interest: having access to defined nanoscale structures is essential to uncovering their intrinsic properties unaffected by sample inhomogeneity. Rigorous understanding of the properties of individual nanocrystals enables exploitation of collective properties of 
nanocrystal ensembles, making it possible to design and fabricate novel electronic, magnetic and photonic devices and other functional materials based on these nanostructures.

Colloidal nanocrystals with a semiconductor as the inorganic material-so-called quantum dots-exhibit size tunable band gaps and luminescence energies due to the quantum size effect ${ }^{3}$. This has led to their use as fluorescent biological labels ${ }^{4-6}$, with colloidal quantum dots now widely employed as targeted fluorescent labels in biomedical research applications. Compared to the organic fluorophores that have been used as biological labels previously, quantum dots are extremely bright and do not photo-bleach, and they provide a readily accessible range of colors. Other applications that could benefit from the combination of low-cost processing with solid-state performance include the use of colloidal quantum dots and rods as possible alternatives to semiconductor polymers in light emitting diodes ${ }^{7}$, lasers ${ }^{8}$, and solar cells ${ }^{9}$. The scope for these applications has prompted intensive study of the synthesis of these materials to optimize colloidal semiconductor nanocrystal fabrication. As a result, many new concepts for controlling the size, shape, and connectivity or coupling of colloidal nanocrystals have been developed first for these materials, but a unified set of synthesis control concepts is now also applied to other classes of materials such as metals and metal oxides. These materials will extend the range of potential applications for colloidal nanocrystals to many other areas, including catalysis.

Over the last decade chemists have come to appreciate that from the point of view of synthesis, colloidal inorganic nanocrystals can be viewed as a class of macromolecule, with preparative strategies that are similar in many ways to those employed with artificial organic polymers. For nanocrystals of one to one hundred nanometers diameter, it is possible to define the average and the dispersion of the diameter, as well as the aspect ratio; the degree of precision with which the desired structure is realized is similar to what is achieved with synthetic polymers, where the preparative means at our disposal allows us to define the mean number of monomer units in a polymer and the variance of this number, and to build complex topologies through joining or branching of simpler 
macromolecules. As with artificial polymers, some principles have now emerged, which give us the ability to control the size and shape of colloidal nanocrystals. After more than two decades, impressive progress has been made toward the tailored synthesis of colloidal nanocrystals that have well-defined structures. A large variety of colloidal nanocrystals can now be successfully produced using a number of methods, such as coprecipitation in aqueous phase, templating with reverse micelles, hydrothermal/solvothermal synthesis, and surfactant-controlled growth in a hot organic solvent ${ }^{10,11}$. Although there is still lack of a general approach for fabricating colloidal nanocrystals in a precisely controlled manner, it is widely accepted that the organic surfactants plays a key role in determining not only the size, but also the shape of the products.

Here, we describe a consistent set of concepts for controlling the growth of colloidal inorganic nanocrystals in a hot organic surfactant (Fig. 1). Because successful control depends extensively on using organic surfactants to judiciously manipulate the nanocrystal surfaces, we start with a discussion of the organic-inorganic interface and the possibilities offered by dynamic surface solvation with surfactant molecules. We next introduce the concepts underpinning kinetic control, which are widely employed to achieve narrow nanocrystal size distributions and provide some control over particle shape. We then discuss how kinetic control in conjunction with selective adhesion can provide even finer control over nanocrystal growth, as required for producing more complex shapes. Finally we close the paper by returning to a comparison to organic polymer synthesis, in order to point important issues that still need to be addressed as well as some exciting opportunities that remain open in the area of colloidal nanocrystal synthesis.

\section{General synthesis scheme}

A typical synthesis system for colloidal nanocrystals consists of three components: precursors, organic surfactants, and solvents. In some cases, surfactants also serve as solvents. Upon heating a reaction medium to a sufficiently high temperature, the 
precursors chemically transform into active atomic or molecular species (monomers); these then form nanocrystals whose subsequent growth is greatly affected by the presence of surfactant molecules. The formation of the nanocrystals involves two steps: nucleation of an initial 'seed', and subsequent growth. In the nucleation step, precursors decompose or react at a relatively high temperature to form a supersaturation of monomers followed by a burst of nucleation of nanocrystals. These nuclei then grow further by consuming additional monomers.

This thermolysis approach will generate nanoparticles; but these particles are crystalline solids only if the atoms can rearrange and anneal during growth, which requires overcoming thermal barriers. The cohesive energy per atom, correlating with the melting temperature of the solid, is therefore a decisive factor in determining optimal conditions for nanocrystal growth. The first step then in colloidal nanocrystal synthesis is to choose a temperature for growth that is hot enough to allow rearrangement of atoms and annealing within a growing nanocrystal over the course of the synthesis. A great benefit in this regard is the reduction in melting temperatures for crystals of smaller size. This effect is the subject of one of the most famous and well-documented scaling laws for the properties of solids in the nanometer regime ${ }^{12}$, and is driven by the fact that in the nanoscale regime, the liquid phase has lower surface energy than a solid with facets, edges, and corners. The effect can be quite significant, leading to a halving of the melting temperature for a solid particle of 2 to $3 \mathrm{~nm}$ diameter, relative to that of the corresponding bulk solid.

The large reduction in melting temperature greatly increases the range of inorganic colloidal nanocrystals that can be grown at temperatures where common organic molecules are stable, which is in the range of $200-400^{\circ} \mathrm{C}$. In fact, the desire to extend colloidal nanocrystals synthesis to the widest possible range of materials has focused much interest on growth at these highest possible temperatures. Organic surfactant molecules in the growth medium are chosen for their propensity to adhere to a growing crystal. Since up to half the atoms making up a nanocrystal may be on its surface, this completely alters the growth strategy to the point where the organic- 
inorganic interface becomes pivotal. In this respect, colloidal nanocrystal growth is strongly related to the field of bio-mineralization, where complex patterns of biologically organized organic functionalities have the effect of controlling the size, shape, and spatial arrangement of some inorganic solids ${ }^{13,14}$. However, the common bio-minerals produced in this way are strongly ionic solids, with cohesive energies that allow growth at room temperature and in water.

As recognized by Steigerwald ${ }^{15,16}$, an important step to the generation of colloidal inorganic nanocrystals is identifying suitable precursor molecules such as organometallic compounds: they need to rapidly decompose or react at the required growth temperature to yield reactive atomic or molecular species (the monomers) that can then contribute to nanocrystal nucleation and growth. The most famous example is the use of dimethyl cadmium and trialkyl phosphine selenide to yield CdSe. Injection of these precursors into a hot solution can yield supersaturation, nucleation, and subsequent growth. The most successfully employed precursors have been relatively simple molecules with "leaving groups" that readily depart to leave behind the desired reactive species. This is somewhat distinct from the precursors employed in Chemical Vapor Deposition (CVD) processes in high vacuum, where the growth temperature may be much higher. Still, the two approaches share many features such as the similar chemical reactions, and a literature review of CVD precursors is often a good starting place for finding a new pathway to making a colloidal nanocrystal. In colloidal solution the true microscopic mechanism of monomer addition is often still not well understood, owing to the complexity of the growth medium. However, the crystal growth kinetics can be qualitatively understood using crystal growth concepts and assuming of a population of nanocrystals in the presence of a solution of active monomers.

\section{Organic-inorganic interface and dynamic solvation}

Surfactant coated nanocrystals in which an inorganic core is surrounded by a "monolayer" of organic molecules hold the potential for the creation of new 
materials ${ }^{17,18}$. The possibility of combining the physical properties of inorganic solids with the low-cost high-volume processing of plastics provides a major impetus for this research $^{19}$. Because the organic-inorganic interface present in these systems holds the key to the synthesis of far more advanced materials, the interface itself is also attracting growing interest.

The energy with which surfactant molecules present in the growth medium adhere to the surfaces of growing nanocrystals is one of the most important parameters influencing crystal growth. The adhesion energy needs to be such that it allows dynamic solvation at the growth temperature: the organic molecule acting as a surfactant needs to be able to exchange on and off the growing crystals, such that regions of the nanocrystal surface are transiently accessible for growth, yet entire crystals are on average monolayer protected to block aggregation. The classic paper of Murray, Norris, and Bawendi ${ }^{20}$ introduced and demonstrated this concept for the growth of CdSe nanocrystals in trioctylphosphine oxide.

Examples of organic surfactants that dynamically solvate nanocrystals include alkyl phosphine oxides, alkyl phosphonic acids, alkyl phosphines, fatty acids and amines, and some nitrogen-containing aromatics. These molecules all contain metal coordinating groups as well as solvophilic groups. The metal coordinating groups are typically electron-donating to allow coordination to electron-poor metal atoms at the nanocrystal surface, thus preventing further growth and aggregation. The other end of the surfactant molecule extends to the solvent and therefore determines the solubility of the nanocrystals; in most cases, it provides the particles with a hydrophobic surface. As of this writing, there is no generally accepted experimental or theoretical method for determining the adhesion energy of an organic surfactant on a nanocrystal surface, so the choice of surfactant remains empirical. This makes screening techniques borrowed from biochemistry promising tools for discovery of appropriate surfactant systems ${ }^{21}$. In all cases, great care must be taken to carefully examine the purity of the organic surfactants and their thermal stability: in numerous instances small percentages of organic impurities were found to play a key role in the growth kinetics. For example, phosphonic acids were 
only recognized as essential ingredients for shape control of CdSe and other II-VI nanocrystals ${ }^{22}$ after their presence in trioctylphosphine oxide was finally controlled.

As temperature decreases, surfactant molecules are less likely to leave the nanocrystal surface. But dynamic solvation can also be achieved at room temperature. For instance, if CdSe nanocrystals coated with TOPO are refluxed in pyridine, the more weakly adherent but more abundant pyridine displaces the TOPO by mass action. Owing to its inherently weaker adhesion energy, pyridine dynamically solvates CdSe even at room temperature so that nanocrystals deposited from pyridine and placed in vacuum display UHV clean surfaces ${ }^{23}$. Surfactants exchange to introduce other competing ligands also allows further derivatization of the nanocrystal surface to introduce a wide range of possible chemical functionalities. This strategy provides an additional means for chemical manipulation of the physical nanocrystal properties, which tend to be sensitive to the nature of the surface coating ${ }^{23}$. It also provides various means to link the nanocrystals to other surfaces and biomolecules ${ }^{24,25}$.

The surfactant molecules not only bind to the growing nanocrystal surface, but also complex the reactive monomer species produced upon heating. The stability and diffusion rate of these complexes, as well as the binding strength of the surfactant molecules to the growing nanocrystal surface are all strongly temperature dependent. Increasing the temperature greatly decreases the stability of the intermediate complexes formed in solution and the binding strength of the surfactants to the nanocrystal surface, while at the same time increasing the diffusion rates of the complexes; this favours the nucleation and growth of the nanocrystals. However, too high a temperature may also lead to uncontrolled growth so that it is impossible to exploit subtle kinetic or energetic effects to achieve precise control over the size and size distribution of the nanocrystals. Choosing an appropriate temperature range is therefore one of the key steps in obtaining control over nanocrystal growth.

\section{Kinetic size control}


The preparation of nanocrystals with relatively narrow size distribution is also a key feature of many modern preparation methods ${ }^{27}$. To understand the concepts at work here, consider a simple model of growth rate versus nanocrystal radius as shown in Fig. 2. The dependence of the surface energy on size explains the left hand side of the curve: very small crystals are unstable due to their large fraction of active surface atoms, as indicated by the negative growth rate. The right hand side of the curve illustrates that larger crystals with smaller surface to volume ratio are stable and grow. The zerocrossing point occurs at the critical size, where nanocrystals neither grow nor shrink. The critical size depends on the monomer concentration, with low monomer concentration favoring a larger critical size. The peak in growth rate versus radius on the right hand side arises due to a geometric factor: compared to a relatively small crystal, many more atoms need to be incorporated to increase the radius of crystals as they become larger.

These considerations explain why a slow growth rate, which produces equilibrated and nearly round crystals, also yields very broad size distributions. Slow growth rates imply low monomer concentration and a high likelihood that the critical size will lie within the distribution of nanocrystal sizes present (Fig. 2). The resultant Ostwald ripening — small crystals will shrink while large ones will grow — then leads to a broad, skewed size distribution. It is still possible to recover a monodisperse sample by separating out fractions of particles with a narrow distribution from the original broadly distributed sample, using one of several separation techniques. Of these, "size selective precipitation” is the most generally applicable method ${ }^{20,28}$. It involves step-wise addition of a poor solvent to a stable solution of nanocrystals to gradually reduce the solvating power, so that larger nanocrystals, with greater attractive van der Waals or dipolar forces between them, precipitate out first. This approach produces nanocrystal fractions with narrow size distributions, but can be time-consuming, tedious, and yields small quantities of the desired material. Further, it only works well with round crystals since the attractive forces between anisotropic nanocrystals depend on multiple parameters ${ }^{29}$.

A more robust approach to obtaining narrow distributions uses the concept of "size distribution focusing” (Fig. 2), based on the prediction of Howard Reiss of more than 50 
years ago that small crystals will grow more rapidly than larger ones if monomer concentrations are sufficiently high ${ }^{30}$. Consider the slow growth conditions described above, and imagine that the monomer concentration is abruptly increased by a secondary injection of precursor. Immediately after injection, the distribution of nanocrystal sizes present has not yet changed; but the critical size, which depends on monomer concentration, shifts to a smaller value. If this shift is large enough, the entire distribution of sizes will now lie on the falling side of the growth vs. radius curve (i.e., all nanocrystals are larger than the size for which the growth rate peaks), and the distribution will spontaneously narrow or "focus." The concept of size distribution focusing has now been clearly demonstrated experimentally ${ }^{31}$. Results are optimized if the monomer concentration is kept such that the average nanocrystal size present is always slightly larger than the critical size. When the monomer concentration is depleted due to growth, the critical size becomes larger than the average size present, and the distribution broadens as the result of Ostwald ripening. Judicious replenishment of monomer can thus be an important feature of the synthesis strategy. Focusing has the advantage that it can produce large quantities of crystals with a narrow size distribution, providing the reaction can be arrested in the appropriate regime (Fig. 3). It is a key first step in kinetic control over nanocrystal synthesis, and we have found that when focusing can be achieved, it opens the door to realize kinetic shape control.

In general, it is desirable for the nucleation event to be separated in time from the growth step in order to obtain relatively monodisperse samples. This means that nucleation must occur on a short time scale. This may be achieved by rapidly injecting suitable precursors into the solvent at high temperature, so as to generate transient supersaturation in monomers and hence induce a nucleation burst. A rapid and intense nucleation burst will lower the monomer concentration below the nucleation threshold, so monomers remaining in solution will only add to the existing nuclei. In many cases, there is some overlap between the nucleation and growth time sales, so the resultant dispersion in nanocrystal sizes needs to be compensated for with focusing. But in optimal cases, it is possible to remain in the fast growth focusing regime while remaining below the nucleation limit. 
Interestingly, rapid injection of precursor does not always lead to quick nucleation. For example, during the synthesis of iron oxide nanocrystals, the injection of the precursor of iron pentacarbonyl is followed by a long incubation time before a sudden burst of nucleation takes place (M. F. Casula et al., unpublished data). This “delayed nucleation” is due to the gradual transformation of iron pentacarbonyl into intermediate species (such as higher nuclearity clusters of carbonyls or metal-surfactant complexes), which then serve as the active "monomer" species during crystal growth. Since nucleation depends exponentially on the monomer concentration, when the nucleation threshold is surpassed, a brief spurt of nucleation occurs. The nucleation event depletes the monomer, and growth follows with no further nucleation. Delayed nucleation is extremely useful because it removes the need for a rapid (and in many cases irreproducible) initial injection of precursor.

\section{Kinetic shape control}

Compared to equilibrium nanocrystals with nearly "round” shapes, nanocrystals with highly anisotropic shapes have larger surface areas that render them metastable, high-energy forms. Formation of the metastable nanocrystals thus requires a kinetic growth regime, whereas equilibrium nanocrystals with low aspect ratios are obtained in the slow growth limit under thermodynamic control. At low growth rate, nearly round nanocrystals are formed, with broad size distribution. At higher growth rate, focusing is observed. When the growth rate is increased just beyond the focusing regime, an astonishing variety of highly anisotropic shapes are obtained, starting with simple rods and disks, but ultimately including shapes like arrows and tetrapods.

The equilibrium shape of inorganic nanocrystals, although faceted, has a low aspect ratio both because this minimizes surface area, and because the low-energy facets of the crystal are relatively close to each other in energy. However, the growth rate of a crystal facet depends exponentially on the surface energy, so that at high growth rates, in a kinetically controlled growth regime, high-energy facets will grow more quickly than 
low-energy facets (Fig. 1a). The progression from Ostwald ripening to focusing to kinetic shape control was first seen in colloidal CdSe nanocrystals ${ }^{32}$, but subsequently has been observed in a variety of other systems, such as cobalt ${ }^{33,34}$ and titanium dioxide ${ }^{35}$.

The onset of kinetic shape control can be widely adjusted using the method of selective adhesion (Fig. 1b). According to the concept of dynamic solvation, organic surfactants are exchanging on the nanocrystals surface during growth. In a faceted crystal, however, the exchange rate on the different facets need not be the same. The introduction of an organic molecule that selectively adheres to a particular crystal facet can be used to effectively lower the energy and hence slow the growth rate of that facet relative to others (Fig. 4). It is more practical to adjust the relative growth rates than it is to increase the absolute rates to the point where the variations are significant. Selective adhesion effects have not been observed directly during nanocrystal growth, but theoretical studies lend credence to the concept ${ }^{36,37}$. However, Peng has suggested an alternative mechanism that involves complexation of the reactive monomer species in solution by organic molecules; this leads to an environment with high chemical potential and can be used to adjust relative growth rates ${ }^{38}$.

In the kinetic growth regime, it is possible to create sequences of events that produce more intricate shapes. A first example is the remarkable phenomenon of sequential elimination of a high-energy facet ${ }^{22}$ (Fig. 1c). It is well known that fast growing facets will eventually disappear during growth, resulting in a crystal terminated by slower growing facets. Consider the possibility that the relative growth rates of two different low index facets differ greatly. In that case, the higher energy facet will grow so quickly that a second or even third layer of atoms can start to form before a first layer is complete. The possibility exists that there is another facet, intermediate in energy between the low and high energy ones present initially. Such a facet may form transiently during the growth of the high-energy facet. Once such a facet forms, it will persist, replacing the initial high-energy facet. This new intermediate energy facet will still grow more quickly than the initial slow growing one, so that the shape will evolve in a 
complex pattern during growth. This has been used to form arrow shaped nanocrystals of $\mathrm{CdSe}^{22}$ and zigzag shaped crystals of $\mathrm{TiO}_{2}{ }^{35}$ (Fig. 5).

A different but related approach to the creation of nanocrystals with complex shapes and connectivity is "oriented attachment" ${ }^{39}$. This remarkable process, first described for $\mathrm{TiO}_{2}$ by Penn and Banfield, involves the coalescence of faceted nanocrystals in such a way as to eliminate two high energy facets ${ }^{40-42}$. The detailed mechanism of oriented attachment remains unclear, but the process seems to occur for many materials systems. The most frequent products of oriented attachment are rods and wires. The signature of this mechanism is the observation of primary particles with the same diameter as the final one-dimensional product, as well as the growth of rods by "quantum units" of the size of a primary nanocrystal. The degree and nature of the attachment process can be manipulated by surfactant control. Indeed, specific chemical transformation of the surfactant on high-energy facets may already play a role in some documented cases $^{43-44}$. Banfield has shown that many defects in natural minerals may have arisen through the oriented attachment process ${ }^{45}$.

Another sequence of events results in controlled branching of colloidal nanocrystals ${ }^{46}$ (Fig. 1d). Branched crystals of zinc oxide were originally discovered in smokes from zinc smelting plants and have been prepared and studied in CVD systems for several decades ${ }^{47}$. In colloidal systems, it is now possible to prepare ensembles of centrally branched tetrapod nanocrystals with a high degree of control over the branch length and diameter ${ }^{48,49}$. Polytypism ${ }^{50}$, or the existence of two or more crystal structures in different regions of the same crystal, coupled with the manipulation of surface energy at the nanoscale, is exploited to controllably produce the branched inorganic nanostructures. In the case of the II-VI semiconductors such as CdSe, the cubic zincblende $\mathrm{ABC}$ stacking of planes is slightly higher in energy but kinetically favored over the hexagonal wurtzite $A B A B$ stacking. Upon injection of precursors, the high concentration of monomer favors nucleation of a pyramidal seed with a zincblende structure; this seed shares a common crystal facet—-the (111) facet— with rod-shaped CdSe with a hexagonal, wurtzite structure. As the monomer concentration drops, the 
(111) facets of the zincblende core switch to ABAB growth in the [1000] direction of the hexagonal phase; this yields a crystalline inorganic structure of four rods at the tetrahedral angle, a so-called tetrapod (Figs. 6a,b). The presence of a selective adhesion agent that stabilizes the side-walls of the hexagonal rods relative to the (111) facet of the zincblende phase is a likely reason for the ability to produce these structures with uniformity and control. Most recently, a wide variety of such inorganic dendrimers with branch points at defined locations on rods and tetrapods have been prepared as well ${ }^{51}$ (Figs. 6c,d). The subsequent branch points can be created by kinetically driving the reaction again, or by nucleation of a second material, such as CdSe on CdTe. These dendritic heterostructures are the most complex structures produced to date in colloidal nanocrystal synthesis.

\section{The outlook}

The close topological similarity between inorganic dendrimers and organic dendrimers helps to emphasize the close similarity between organic polymers and inorganic nanocrystals in general from the point of view of chemistry. Will it ever be possible to create inorganic nanocrystals with the varied and rich compositional and spatial complexity of organic systems? Inorganic nanocrystals can already be created with far more complex shapes and with far greater control over size and shape than had long been thought possible. Colloidal nanocrystals are about the same size as an organic macromolecule, and with organic surfactants on the inorganic nanocrystal surface, they can be manipulated in much the same way as organic polymers ${ }^{52}$. The fact that solid-state materials can be manipulated chemically in such similar ways to polymers has led to a near explosion of work in organic-inorganic colloidal nanocrystals.

At the same time, as of this writing, we are still far from a quantitative description of how organic molecules bind and pack on nanocrystal surfaces. We have the qualitative phenomenology and general framework for kinetic shape control described here. However, the next stage requires specific knowledge of selective surface adhesion energies on nanocrystals, including the dependence of adhesion energy on coverage and co-adhesion. At a minimum, this will require combinations of surface science model 
experiments, quantum theoretical calculations, and the development of new synchrotron based surface structural analysis on colloidal nanocrystals. Improved experimental studies of nanocrystal growth kinetics, including spectroscopic identification of "monomers," and real time monitoring of average size and shape are very much needed. There is much hope that microfluidics will help in this regard, enabling rapid temperature jump, and control of concentration versus time, as well as on-chip monitoring and analysis $^{53}$. The long-term goal is the preparation of inorganic crystals with even greater degrees of shape and compositional complexity. In the condensed matter physics community, it is common to speak of nanocrystals as "artificial atoms", with controlled density of states and designed properties ${ }^{54}$. Extending this analogy, we can imagine the possibility of creating "artificial molecules" consisting of groups of interconnected inorganic nanocrystals of controlled size, shape, and three-dimensional orientation ${ }^{27,55-62}$. Such systems would have analogous complexity to organic molecules, and may therefore exhibit a remarkable range of new functionalities. Success in this endeavor will require better understanding and control of the organic-inorganic interface.

\section{Reference:}

1. Alivisatos, A. P. Nanocrystals: building blocks for modern materials design. Endeavour 21, 56-60 (1997).

2. El-Sayed, M. A. Small is different: shape-, size-, and composition-dependent properties of some colloidal semiconductor nanocrystals. Acc. Chem. Res. 37, 326-333 (2004).

3. Alivisatos, A. P. Perspectives on the physical chemistry of semiconductor nanocrystals. J. Phys. Chem.

100, 13226-13239 (1996).

4. Bruchez, M., Moronne, M., Gin, P., Weiss, S. \& Alivisatos, A.P. Semiconductor nanocrystals as fluorescent biological labels. Science 281, 2013-2016 (1998).

5. Michalet, X., Pinaud, F. F., Bentolila, L. A., Tsay, J. M., Doose, S., Li, J. J., Sundaresan, G., Wu, A. M., Gambhir, S. S. \& Weiss, S. Quantum dots for live cells, in vivo imaging, and diagnostics. Science 307, 538-544 (2005).

6. Gu, W., Larabell, C. \& Alivisatos, A. P. Quantum dots as cellular probes, Annu. Rev. Biomed. Eng. in press.

7. Tessler, N., Medvedev, V., Kazes, M., Kan, S., Banin, U. Efficient near-infrared polymer nanocrystal light-emitting diodes. Science 295, 1506-1508 (2002).

8. Kazes, M., Lewis, D. Y., Ebenstein, Y., Mokari, T. \& Banin, U. Lasing from semiconductor quantum rods in a cylindrical microcavity. Adv. Mater. 14, 317-321 (2002). 
9. Huynh, W. U., Dittmer, J. J. \& Alivisatos, A. P. Hybrid nanorod-polymer solar cells. Science 29, 24252427 (2002).

10. Cushing, B. L., Kolesnichenko, V. L. \& O’Connor, C. J. Recent advances in the liquid-phase syntheses of inorganic nanoparticles. Chem. Rev. 104, 3893-3946 (2004).

11. Pileni, M. P. The role of soft colloidal templates in controlling the size and shape of inorganic nanocrystals. Nature Mater. 2, 145-150 (2003).

12. Buffat, Ph. \& Borel, J-P. Size effect on the melting temperature of gold particles. Phys. Rev. A 13, 2287-2298 (1976).

13. Mann, S. Molecular recognition in biomineralization, Nature 332, 119-124 (1988).

14. Bianconi, P. A., Lin, J. \& Strzelecki, A. R. Crystallization of an inorganic phase controlled by a polymer matrix. Nature 349, 315-317 (1991).

15. Stuczynski, S. M., Brennan, J. G. \& Steigerwald M. L. Formation of metal-chalcogen bonds by the reaction of metal-alkyls with silyl chalcogenides, Inorg. Chem. 28, 4431-4432 (1989).

16. Steigerwald, M. L. Clusters as small solids. Polyhedron 13,1245-1252 (1994).

17. Nirmal, M. \& Brus, L. Luminescence photophysics in semiconductor nanocrystals. Acc. Chem. Res. 32, 407-414 (1999).

18. Alivisatos, A. P. Semiconductor clusters, nanocrystals, and quantum dots. Science 271, 933-937 (1996).

19. Steckel, J. S., Coe-sullivan, S., Bulovic, V. \& Bawendi, M. G. $1.3 \mu \mathrm{m}$ to $1.55 \mu \mathrm{m}$ tunable electroluminescence from PbSe quantum dots embedded within an organic device. Adv. Mater. 15, 18621866 (2003).

20. Murray, C. B., Norris, D. J. \& Bawendi, M. G. Synthesis and characterization of nearly monodisperse $\mathrm{CdE}$ (E = sulfur, selenium, tellurium) semiconductor nanocrystallites. J. Am. Chem. Soc. 115, 8706-8715 (1993).

21. Whaley, S. R., English, D. S., Hu, E. L., Barbara, P. F. \& Belcher, A. M. Selection of peptides with semiconductor binding specificity for directed nanocrystal assembly. Nature 405, 665-668 (2000).

22. Manna, L., Scher, E. C., \& Alivisatos, A. P. Synthesis of soluble and processable rod-, arrow-, teardrop-, and tetrapod-shaped CdSe nanocrystals. J. Am. Chem. Soc. 122, 12700-12706 (2000).

23. Katari, J. E. B., Colvin, V. L. \& Alivisatos, A. P. X-ray photoelectron spectroscopy of CdSe nanocrystals with applications to studies of the nanocrystal surface. J. Phys. Chem. 98, 4109 - 4117 (1994). 24. Kuno, M., Lee, J. K., Dabbousi, B. O., Mikulec, F. V. \& Bawendi, M. G. The band edge luminescence of surface modified CdSe nanocrystallites: probing the luminescing state. J. Chem. Phys. 106, 9869-9882 (1997).

25. Klein, D. L., Roth, R., Lim, A. K. L., Alivisatos A. P. \& McEuen, P. L. A single-electron transistor made from a cadmium selenide nanocrystal. Nature 389, 699-701 (1997).

26. Chan, W. C. \& Nie, S. Quantum dot bioconjugates for ultrasensitive nonisotopic detection. Science 281, 2016-2018 (1998).

27. Redl, F. X., Cho K.-S., Murray, C. B. \& O’Brien S. Three-dimensional binary superlattices of magnetic 
nanocrystals and semiconductor quantum dots. Nature 423, 968-971 (2003).

28. Vossmeyer, T., Katsikas, L., Giersig, M., Popovic, I. G., Diesner, K., Chemseddine, A., Eychmueller, A. \& Weller, H. CdS nanoclusters: synthesis, characterization, size dependent oscillator strength, temperature shift of the excitonic transition energy, and reversible absorbance shift. J. Phys. Chem. 98, 7665-7673 (1994).

29. Murray, C. B., Sun, S., Doyle, H. \& Betley, T. Monodisperse 3d transition-metal (Co, Ni, Fe) nanoparticles and their assembly into nanoparticle superlattices. MRS Bull. 26, 985-991 (2001).

30. Reiss, H. The growth of uniform colloidal dispersions. J. Chem. Phys. 19, $482-487$ (1951).

31. Peng, X., Wickham, J. \& Alivisatos, A.P. Kinetics of II-VI and III-V colloidal semiconductor nanocrystal growth: focusing, of size distributions. J. Am. Chem. Soc. 120, 5343-5344 (1998).

32. Peng, X., Manna, L., Yang, W., Wickham, J., Scher, E., Kadavanich, A. \& Alivisatos, A. P. Shape control of CdSe nanocrystals, Nature 404, 59-61 (2000).

33. Puntes, V. F., Krishnan, K. M., Alivisatos, A. P. Colloidal nanocrystal shape and size control: the case of cobalt. Science 291, 2115-2117 (2001).

34. Puntes, V. F., Zanchet, D., Erdonmez, C. K. \& Alivisatos A. P. Synthesis of hcp-Co nanodisks. J. Am. Chem. Soc. 124, 12874-12880 (2002).

35. Jun, Y.-W., Casula, M. F., Sim, J.-H., Kim, S. Y., Cheon, J. \& Alivisatos, A. P. Surfactant-assisted elimination of a high energy facet as a means of controlling the shapes of $\mathrm{TiO}_{2}$ nanocrystals. J. Am. Chem. Soc. 125, 15981-15985 (2003).

36. Puzder, A., Williamson, A. J., Zaitseva, N., Galli, G., Manna, L. \& Alivisatos, A. P. The effect of organic ligand binding on the growth of CdSe nanoparticles probed by ab initio calculations. Nano Lett. 4, 2361-2365 (2004).

37. Manna, L., Wang, L. W., Cingolani, R. \& Alivisatos, A. P. First-principles modeling of unpassivated and surfactant-passivated bulk facets of wurtzite cdse: a model system for studying the anisotropic growth of CdSe nanocrystals. J. Phys. Chem. B. 109, 6183-6192 (2005).

38. Yu, W. W., Wang, Y. A. \& Peng, X. Formation and stability of size-, shape-, and structure-controlled CdTe nanocrystals: ligand effects on monomers and nanocrystals. Chem. Mater. 15, 4300-4308 (2003). 39. Alivisatos, A. P. Naturally aligned nanocrystals. Science 289, 736-737 (2000).

40. Penn, R. L. \& Banfield, J. F. Morphology development and crystal growth in nanocrystalline aggregates under hydrothermal conditions: insights from titania. Geochim. Cosmochim. Acta 63, 1549-1557 (1999).

41. Penn, R. L. \& Banfield, J. F. Oriented attachment and growth, twinning, polytypism, and formation of metastable phases: insights from nanocrystalline $\mathrm{TiO}_{2}$. Am. Mineral. 83, 1077-1082 (1998).

42. Pacholski,C., Kornowski, A. \& Weller, H. Self-assembly of ZnO: from nanodots to nanorods. Angew. Chem. Int. Ed. 41, 1188-1191 (2002). 
43. Yu, J. H., Joo, J., Park, H. M., Baik, S-I., Kim, Y. W., Kim, S. C. \& Hyeon, T. Synthesis of quantumsized cubic ZnS nanorods by the oriented attachment mechanism. J. Am. Chem. Soc. 127, 5662-5670 (2005).

44. Adachi, M., Murata, Y., Takao, J., Jiu, J., Sakamoto, M. \& Wang, F. Highly efficient dye-sensitized solar cells with a titania thin-film electrode composed of a network structure of single-crystal-like $\mathrm{TiO}_{2}$ nanowires made by the "oriented attachment" mechanism. J. Am. Chem. Soc. 126, 14943-14949 (2004). 45. Banfield, J. F. \& Penn, R. L. Imperfect oriented attachment: dislocation generation in defect-free nanocrystals. Science 281, 969-971 (1998).

46. Wang, D. \& Lieber, C. M. Nanocrystals branch out. Nature Mater. 2, 355-356 (2003).

47. Yan, H., He, R., Pham, J. \& Yang P. Morphogenesis of one-dimensional ZnO nano- and microcrystals. Adv. Mater. 15, 402-405 (2003).

48. Manna, L., Milliron, D. J., Meisel, A., Scher, E. C. \& Alivisatos, A. P. Controlled growth of tetrapod branched inorganic nanocrystals. Nature Mater. 2, 382-385 (2003).

49. Peng, X. Mechanisms for the shape-control and shape-evolution of colloidal semiconductor nanocrystals. Adv. Mater. 15, 459-463 (2003).

50. Yeh, C. Y., Lu, Z. W., Froyen, S. \& Zunger, A. Zinc-blende-wurtzite polytypism in semiconductors. Phys. Rev. B 46, 10086-10097 (1992).

51. Milliron, D. J., Hughes, S., Cui, Y., Manna, L., Li, J., Wang, L. W. \& Alivisatos, A. P. Colloidal nanocrystal heterostructures with linear and branched topology. Nature 430, 190-195 (2004).

52. Li, L.-S., Walda, J., Manna, L. \& Alivisatos, A. P. Semiconductor nanorod liquid crystals. Nano Lett. 2, 558-560 (2002).

53. Chan, E. M., Mathies, R. A. \& Alivisatos, A. P. Size-controlled growth of CdSe nanocrystals in microfluidic reactors. Nano Lett. 3, 199-201 (2003).

54. Collier, C. P., Vossmeyer, T. \& Heath, J. R. Nanocrystal superlattices, Annu. Rev. Phys. Chem. 49, 371404 (1998).

55. Sun, S., Murray, C. B., Weller, D., Folks, L. \& Moser, A. Monodisperse FePt nanoparticles and ferromagnetic FePt nanocrystal superlattices. Science 287, 1989-1992 (2000).

56. Mirkin, C. A., Letsinger, R. L., Mucic, R. C. \& Storhoff, J. J. A DNA-based method for rationally assembling nanoparticles into macroscopic materials. Nature 382, 607-609 (1996).

57. Alivisatos, A. P., Johnsson K. P., Peng, X., Wilson, T. E., Loweth, C. J., Bruchez, M. P. \& Schultz, P.

G. Organization of 'nanocrystal molecules' using DNA. Nature 382, 609-611 (1996).

58. Fu, A., Micheel, C. M., Cha, J., Chang, H., Yang, H. \& Alivisatos, A. P. Discrete nanostructures of quantum dots/Au with DNA. J. Am. Chem. Soc. 126, 10832-10833 (2004).

59. Yu, H., Chen, M., Rice, P. M., Wang, S. X., White, R. L. \& Sun, S. Dumbbell-like bifunctional Au$\mathrm{Fe}_{3} \mathrm{O}_{4}$ nanoparticles. Nano Lett. 5, 379-382 (2005). 
60. Gu, H., Zheng, R., Zhang, X. \& Xu, B. Facile one-pot synthesis of bifunctional heterodimers of nanoparticles: a conjugate of quantum dot and magnetic nanoparticles. J. Am. Chem. Soc. 126, 5664-5665 (2004).

61. Mokari, T., Rothenberg, E., Popov, I., Costi, R. \& Banin, U. Selective growth of metal tips onto semiconductor quantum rods and tetrapods. Science 304, 1787-1790 (2004).

62. Kudera, S., Carbone, L., Casula, M. F., Cingolani, R., Falqui, A., Snoeck, E., Parak, W. J. \& Manna, L. Selective growth of PbSe on one or both tips of colloidal semiconductor nanorods. Nano Lett. 5, $445-449$ (2005).

\section{Acknowledgements}

The authors acknowledge the support from the Molecular Foundry at the Lawrence Berkeley National Laboratory. 


\section{Figure Captions}

Figure 1 Concepts of shape control of colloidal nanocrystals. a, Kinetic shape control at high growth rate. The high energy facets grow more quickly than low energy facets in a kinetic regime. b, Kinetic shape control by using the method of selective adhesion. The introduction of an organic molecule that selectively adheres to a particular crystal facet can be used to slow the growth of that facet relative to others, leading to the formation of rod or disk shaped nanocrystals. c, More intricate shapes as a result of sequential elimination of a high energy facet. The persistent growth of an intermediate energy facet eventually eliminates the initial high energy facet, forming complex structures such as arrow or zigzag shaped nanocrystals. d, Controlled branching of nanocrystals. The existence of two or more crystal structures in different domains of the same crystal, coupled with the manipulation of surface energy at the nanoscale, can be exploited to produce branched inorganic nanostructures such as tetrapods. Inorganic dendrimers can be further prepared by creating subsequent branch points at the defined locations on the existing nanostructures.

Figure 2 Concept of size distribution focusing. The growth process of nanocrystals could occur in two different modes, depending upon the concentration of the monomer present: "focusing" and "defocusing" of the size distribution. A critical size exists at any given monomer concentration. At a high monomer concentration, the critical size is small so that all the particles grow. In this situation, smaller particles grow faster than the larger ones, and as a result, the size distribution can be focused down to one that is nearly monodisperse. If the monomer concentration is below a critical threshold, small nanocrystals are depleted as larger ones grow and the size distribution broadens, or defocuses. The preparation of nearly monodisperse spherical particles can be achieved by arresting the reaction while it is still in the focusing regime, with a large concentration of monomer still present.

Figure 3 Monodisperse colloidal nanocrystals synthesized under kinetic size control. a, Transmission electron microscopy (TEM) image of CdSe nanocrystals. b, TEM image of 
Co nanocrystals. c, TEM micrograph of an $\mathrm{AB}_{13}$ superlattice of $\gamma-\mathrm{Fe}_{2} \mathrm{O}_{3}$ and $\mathrm{PbSe}$ nanocrystals. The precise control on the size distributions of both nanocrystals allows their self-assembly into ordered three-dimensional superlattices. (Reprinted by permission of from Nature Publishing Group from ref.27) The scale bars are $50 \mathrm{~nm}$.

Figure 4 Anisotropic growth of nanocrystals by kinetic shape control and selective adhesion: a, CdSe nanorods (scale bar is $50 \mathrm{~nm}$ ) and $\mathbf{b}$, Co nanodisks (scale bar is 100 $\mathrm{nm})$. The organic surfactant molecules selectively adhere to one facet of the nanocrystal, allowing the crystal to grow anisotropically to form a rod or disk shape. (a, Reprinted by permission of American Chemical Society from ref.52)

Figure 5 Nanocrystals with complex shapes prepared by sequential elimination of a high energy facet. a, Two dimensional representation and a HRTEM image of an arrow shaped nanocrystal of CdSe. HRTEM characterization shows that each shape of nanocrystal is predominately wurtzite and that the angled facets of the arrows are the (101) faces. The scale bar is $5 \mathrm{~nm}$. Red and blue dots represent Se and Cd atoms, respectively. (Reprinted by permission of American Chemical Society from ref.22) b, Simulated three-dimensional shape and HRTEM analysis of a $\mathrm{TiO}_{2}$ rod. The long axes of the nanocrystals are parallel to the c-axis of the anatase structure, while the nanocrystals are faceted with $\{101\}$ faces along the short axes. Hexagon shapes (the [010] projection of a truncated octagonal bipyramid) truncated with two $\{001\}$ and four $\{101\}$ faces are observed either at the one end or at the center of the nanocrystals. The branched shape is a result of the growth along [101] directions starting from the hexagon shape. The scale bar is $3 \mathrm{~nm}$. (Reprinted by permission of American Chemical Society from ref.35)

Figure 6 Controlled branching of colloidal nanocrystals. a, HRTEM image of a typical tetrapod-shaped CdSe nanocrystal, looking down the [001] direction of one arm. The nuclei is the zincblende structure, with wurtzite arms growing out of each of the four (111) equivalent faces. b, Low magnification TEM image of CdTe tetrapods. The scale bar is $100 \mathrm{~nm}$. c, HRTEM image of a tetrapod that has branches growing out of each arm. There are zincblende layers near the ends of the original arms, and the branches are 
wurtzite with some stacking faults. d, TEM image of branched tetrapods result from nucleation of CdTe zincblende branch points on the end of each arm. The scale bar is 100 nm. (a, c, Reprinted by permission of American Chemical Society from ref.22; b, Reprinted by permission of from Nature Publishing Group from ref.48; d, Reprinted by permission of from Nature Publishing Group from ref.51 ) 
a

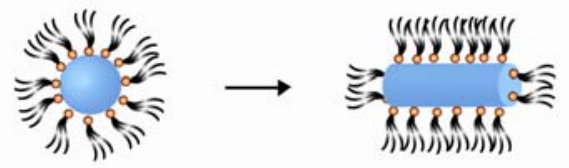

b

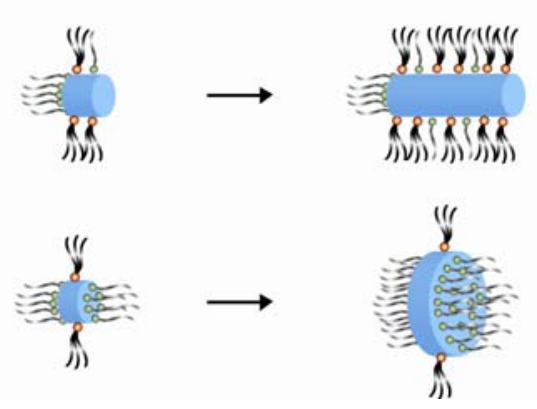

C
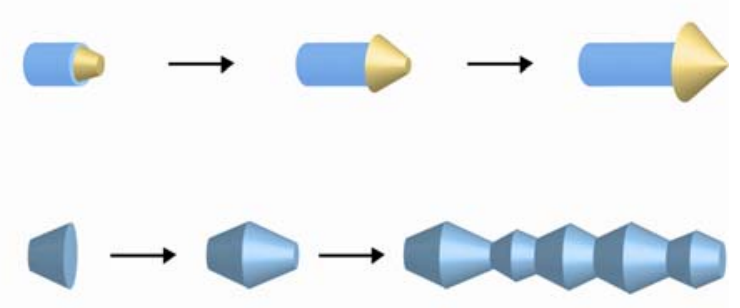

d

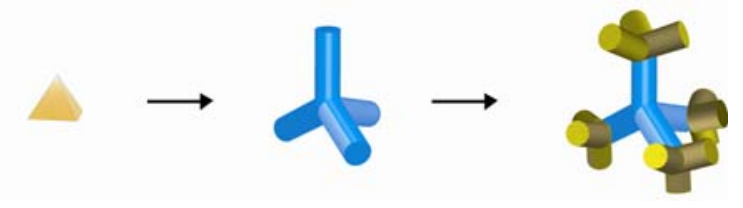

Alivisatos, Figure 1 


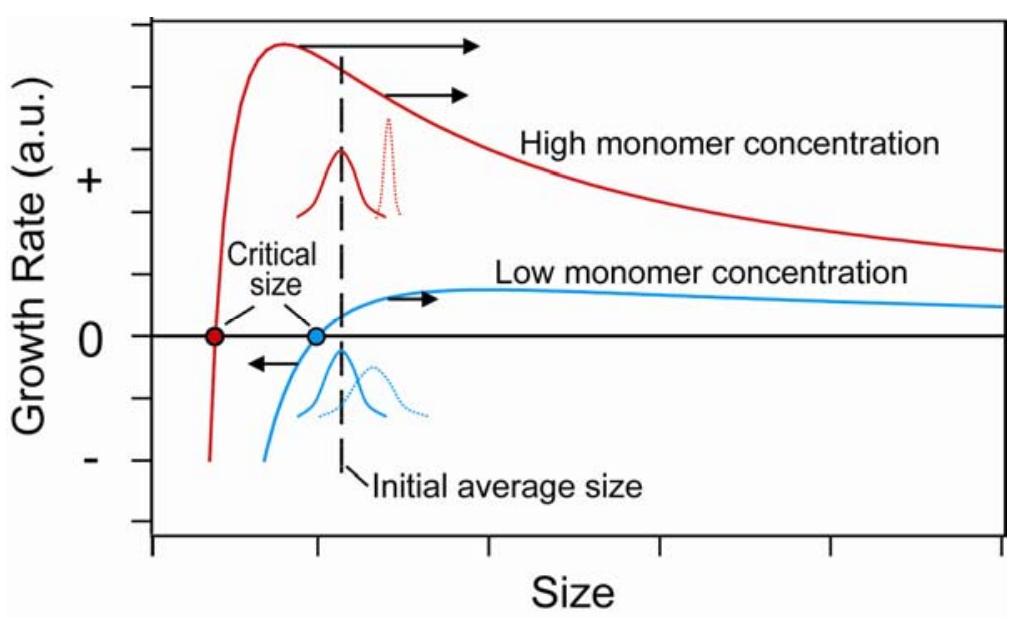

Alivisatos, Figure 2 


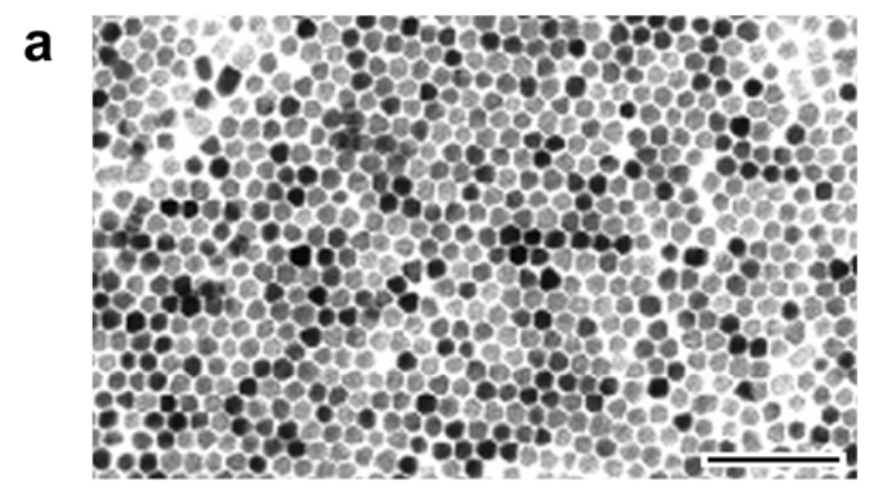

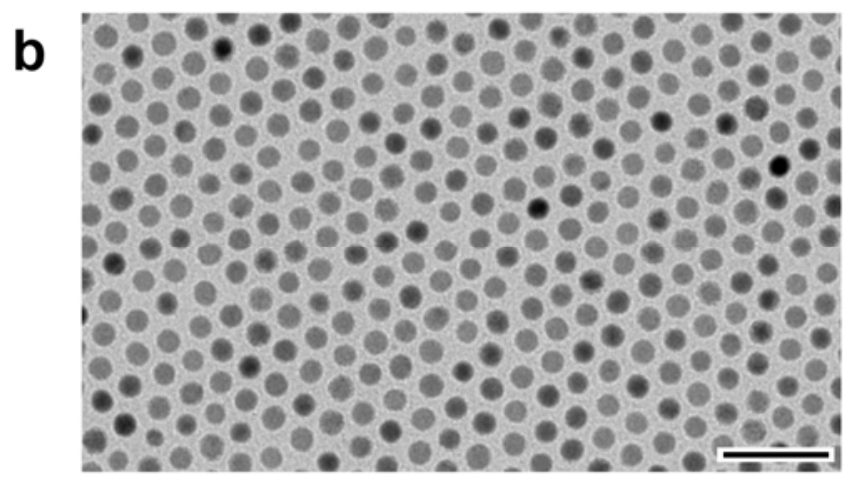

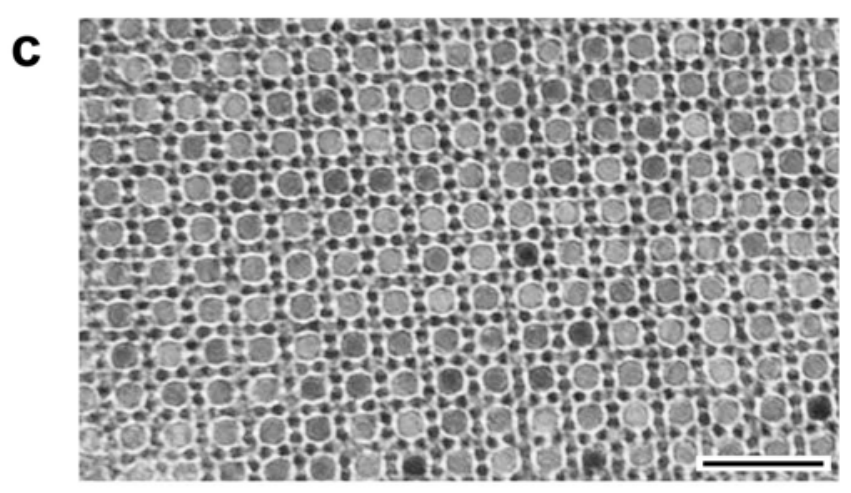

Alivisatos, Figure 3 

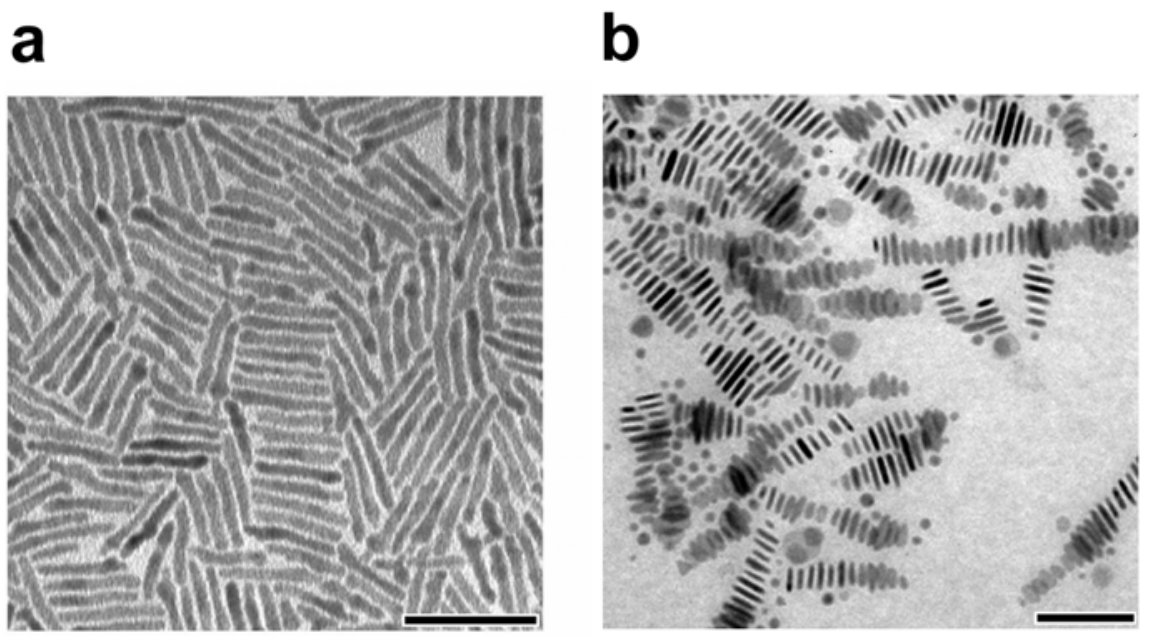

Alivisatos, Figure 4 
a
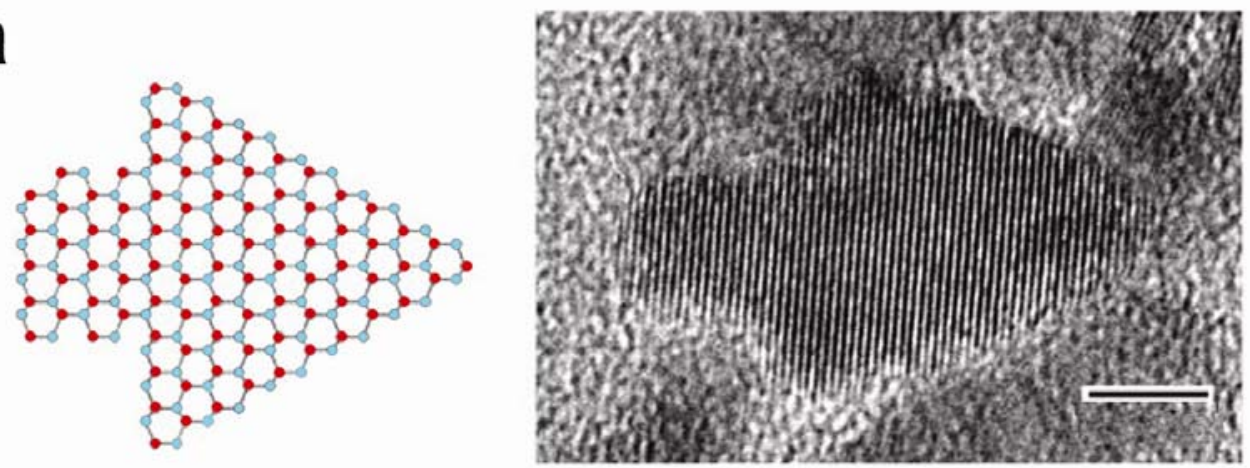

b
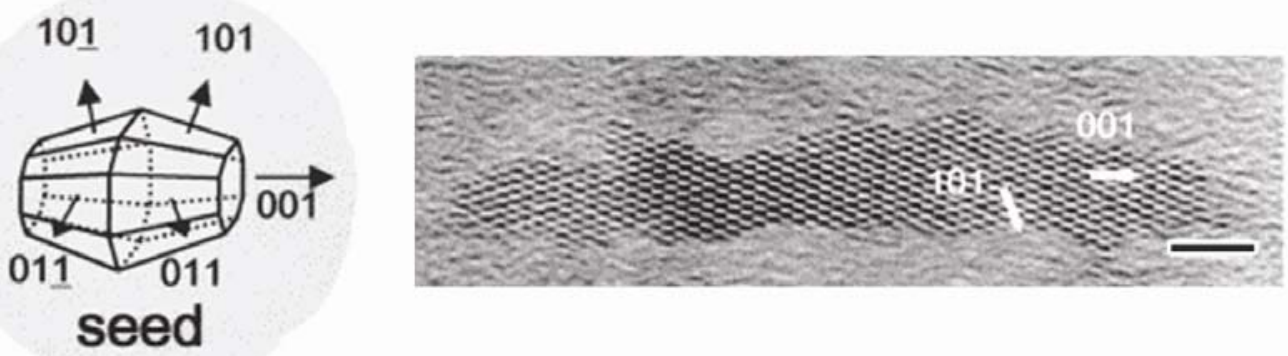

Alivisatos, Figure 5 

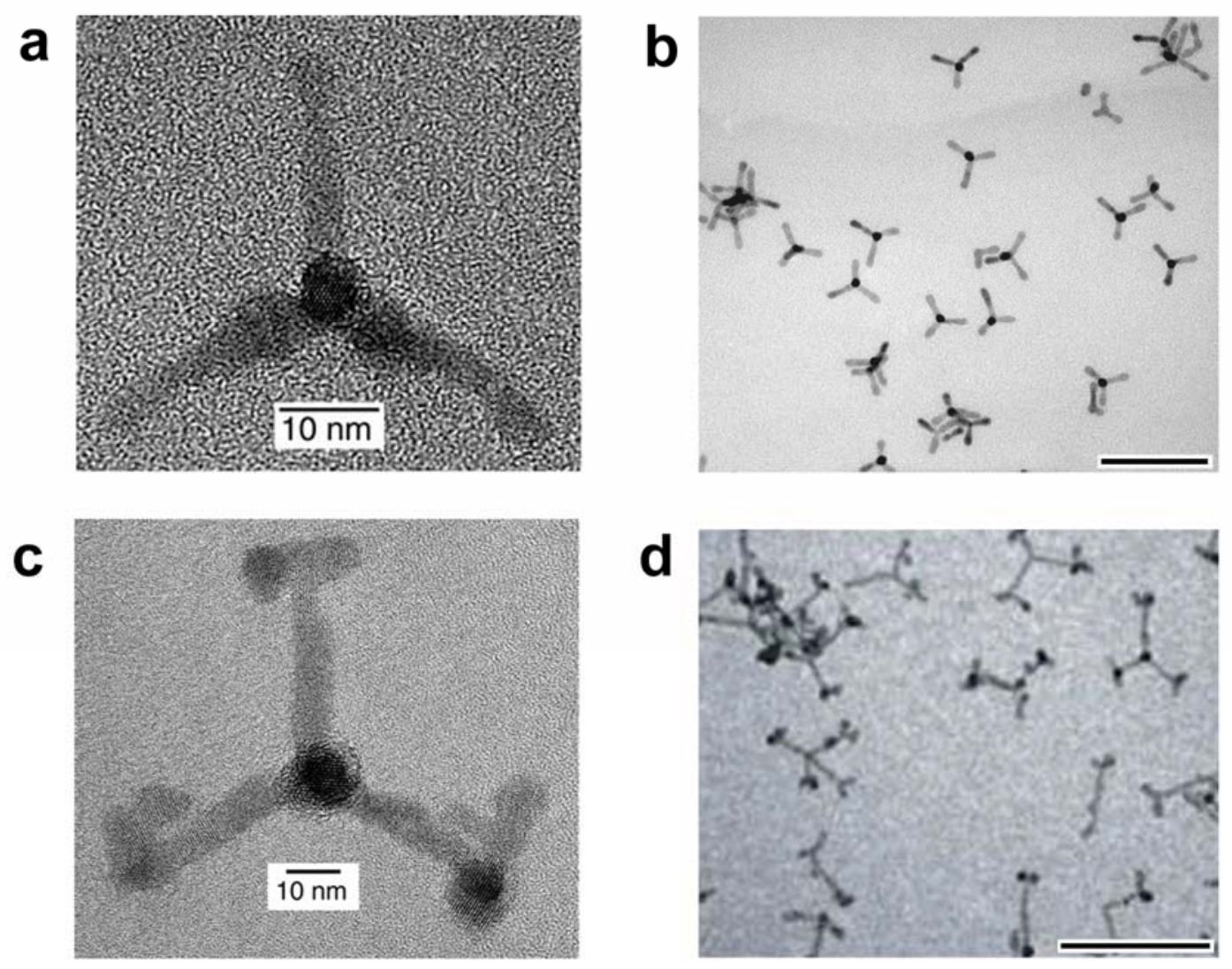

Alivisatos, Figure 6 